\title{
Dimensões nasofaríngeas e sintomas respiratórios após a cirurgia de retalho faríngeo em crianças e adultos
}

\author{
Nasopharyngeal dimensions and respiratory symptoms after \\ pharyngeal flap surgery in children and adults
}

\author{
Ana Paula Fukushiro', Carmen Vivian Domingues Zwicker', Katia Flores Genaro', Renata Paciello Yamashita', \\ Inge Elly Kiemle Trindade ${ }^{1}$
}

\begin{abstract}
RESUMO
Objetivo: Verificar a influência da idade sobre as dimensões nasofaríngeas e os sintomas respiratórios em pacientes submetidos à cirurgia para a correção da insuficiência velofaríngea pela técnica de retalho faríngeo. Métodos: Amostra formada por 103 indivíduos distribuídos em três grupos: crianças (6 a 12 anos), adultos jovens ( 18 a 30 anos) e adultos de meia-idade ( $\geq 40$ anos). Trata-se de estudo retrospectivo que analisou os valores da área nasofaríngea (técnica fluxo-pressão, valor de referência $0,570 \mathrm{~cm}^{2}$ ) e os sintomas de respiração oral, ronco e dificuldade respiratória durante sono, obtidos, em média, 12 meses após a cirurgia. A área nasofaríngea foi comparada entre os grupos por análise de variância e os sintomas respiratórios pelo teste Qui-quadrado $(\mathrm{p}<0,05)$. Resultados: Os valores médios da área nasofaríngea após a cirurgia foram $0,527 \pm 0,215$ $\mathrm{cm}^{2}, 0,599 \pm 0,213 \mathrm{~cm}^{2}$ e $0,488 \pm 0,276 \mathrm{~cm}^{2}$, respectivamente para crianças, adultos jovens e adultos de meia-idade, não havendo diferença entre os grupos. As proporções de respiração oral, ronco e dificuldade respiratória durante o sono foram, respectivamente, $66 \%, 69 \%$ e $9 \%$ nas crianças, $51 \%, 49 \%$ e $7 \%$ nos adultos jovens e $75 \%, 75 \%$ e $19 \%$ nos adultos de meia-idade, com maior frequência de ronco nos adultos de meia-idade. Conclusão: A idade não interferiu nas dimensões nasofaríngeas na presença de retalho faríngeo, mas foi um fator agravante de sintomas respiratórios, principalmente nos indivíduos mais velhos.
\end{abstract}

Descritores: Fissura palatina; Insuficiência velofaríngea; Cirurgia bucal; Sinais e sintomas respiratórios; Rinomanometria

\begin{abstract}
Purpose: To investigate the influence of age on nasopharyngeal dimensions and respiratory symptoms in patients undergoing surgery to correct velopharyngeal insufficiency using the pharyngeal flap technique. Methods: A sample comprising 103 individuals divided into three groups: children (6-12 years old), young adults (18-30 years old) and middle-aged adults ( $\geq 40$ years old). This was a retrospective study that analyzed the values for the nasopharyngeal area (pressure-flow technique, reference value: $0.570 \mathrm{~cm}^{2}$ ) and the symptoms of oral respiration, snoring and respiratory difficulties during sleep. The values were obtained, on average, 12 months after surgery. The nasopharyngeal area was compared between the groups using analysis of variance, and respiratory symptoms were compared using the chi-squared test $(\mathrm{p}<0.05)$. Results: The mean nasopharyngeal area values after surgery were $0.527 \pm 0.215 \mathrm{~cm}^{2}, 0.599 \pm 0.213 \mathrm{~cm}^{2}$ and $0.488 \pm 0.276 \mathrm{~cm}^{2}$ for children, young adults and middle-aged adults, respectively, with no differences between groups. The proportions of oral respiration, snoring and breathing difficulty during sleep were, respectively, 66\%, 69\% and 9\% in children; $51 \%, 49 \%$ and $7 \%$ in young adults; and $75 \%, 75 \%$ and $19 \%$ in middle-aged adults, with a higher frequency of snoring in middle-aged adults. Conclusion: Age did not affect nasopharyngeal dimensions in the presence of a pharyngeal flap, but it was an aggravating factor for respiratory symptoms, especially in older individuals.
\end{abstract}

Keywords: Cleft palate; Velopharyngeal insufficiency; Surgery, oral; Signs and symptoms, Respiratory; Rhinomanometry

Trabalho desenvolvido no Laboratório de Fisiologia do Hospital de Reabilitação de Anomalias Craniofaciais, Universidade de São Paulo - USP - Bauru (SP), Brasil. (1) Programa de Pós-Graduação em Ciências da Reabilitação, Hospital de Reabilitação de Anomalias Craniofaciais, Universidade de São Paulo - USP - São Paulo (SP), Brasil.

(2) Programa de Pós-Graduação (Mestrado) em Ciências da Reabilitação, Hospital de Reabilitação de Anomalias Craniofaciais, Universidade de São Paulo - USP - São Paulo (SP), Brasil.

Conflito de interesses: Não

Contribuição dos autores: $A P F$ autora principal, participação na ideia original do estudo, coleta de dados, análise dos dados e redação do artigo; $C V D Z$ participação na análise estatística dos dados e redação dos resultados; $K F G$ participação na análise estatística e redação do artigo; $R P Y$ participação na coleta de dados e redação do artigo e $I E K$ líder do grupo de pesquisadores, participação na ideia original do estudo e redação do artigo.

Endereço para correspondência: Ana Paula Fukushiro. R. Sílvio Marchione, 3/20, Vila Universitária, Bauru (SP), Brasil, CEP: $17012-900$.

E-mail: anapaulaf@usp.br

Recebido em: 25/4/2012; Aceito em: 5/6/2013 


\section{INTRODUÇÃO}

A fissura labiopalatina é a deformidade congênita que pode se manifestar no lábio, no palato, ou em ambas as estruturas, havendo a necessidade de realização de cirurgias reparadoras para correção dessa anormalidade. A queiloplastia e a palatoplastia são as primeiras cirurgias executadas durante o longo e complexo tratamento que, em conjunto com as demais abordagens terapêuticas, são fundamentais para a reabilitação do paciente ${ }^{(1)}$.

A fissura palatina é considerada a causa mais frequente da insuficiência velofaríngea (IVF), uma alteração estrutural do mecanismo velofaríngeo, cujo sintoma mais comum é a hipernasalidade, associada à emissão nasal de ar e à fraca pressão aérea intraoral ${ }^{(2-4)}$.

É consenso que a palatoplastia deve ter o objetivo funcional de proporcionar condições para que o véu palatino auxilie o mecanismo velofaríngeo. Todavia, em muitos casos, mesmo após a correção cirúrgica primária, os sintomas da IVF persistem, requerendo procedimento secundário ${ }^{(5,6)}$. Assim, a cirurgia que utiliza a técnica de retalho faríngeo de pedículo superior tem sido um dos procedimentos mais adotados para a correção da IVF. A técnica consiste na construção de uma ponte de tecido miomucoso entre o véu palatino e a parede posterior da faringe, delimitada por dois orifícios laterais que permitem a respiração nasal. A finalidade do retalho é criar uma obstrução mecânica à passagem do fluxo aéreo para eliminar a hipernasalidade e a emissão de ar nasal, com aumento da pressão intraoral ${ }^{(7)}$ durante a produção da fala envolvendo sons orais.

Vários estudos comprovaram o grande sucesso da cirurgia de retalho faríngeo na redução ou eliminação de sintomas da IVF sobre a fala, com proporções de sucesso cirúrgico variando entre $25 \%$ e $98 \%{ }^{(8-10)}$. No entanto, o retalho faríngeo também está associado ao comprometimento das vias aéreas superiores, podendo ocasionar hiponasalidade, obstrução nasal crônica, ronco e apneia obstrutiva do sono, sintomas esses que interferem na função respiratória e, consequentemente, na qualidade de $\operatorname{vida}^{(11-14)}$.

Há ainda que se considerar que a fissura labiopalatina determina deformidades nasais que, com frequência, reduzem as dimensões da cavidade nasal ${ }^{(15)} \mathrm{e}$ aumentam a resistência nasal ao fluxo aéreo respiratório. Assim, é possível que, nesses casos, a colocação de um retalho na região da velofaringe seja um fator agravante no comprometimento da permeabilidade nasal ${ }^{(16)}$.

Alguns estudos realizaram um levantamento de sintomas clínicos de obstrução respiratória, com a determinação da área dos orifícios velofaríngeos, utilizando avaliação instrumental para avaliar o efeito da cirurgia de retalho faríngeo sobre a respiração em indivíduos com fissura de palato ${ }^{(14,17,18)}$.

Em amostra preliminar, foi observado que o retalho faríngeo tem efeito variável sobre a via aérea nasal ${ }^{(18)}$, tendo sido encontradas modificações positivas e negativas induzidas pelo retalho. Os autores atribuíram esse resultado à presença de obstrução nasal prévia, encontrada em cinco dos sete pacientes estudados, confirmando que o retalho diminuiu ainda mais a via aérea nasal, o que explicaria o aparecimento de dificuldades respiratórias como ronco, obstrução nasal crônica e apneia obstrutiva do sono.

Ao estudar os distúrbios respiratórios do sono em adultos de meia-idade que haviam realizado cirurgia para a correção da IVF utilizando retalho faríngeo, um estudo observou respiração oral, ronco e episódios de obstrução respiratória em 53\% dos casos, confirmando que o retalho faríngeo pode contribuir para o agravamento dos distúrbios respiratórios nessa faixa etária ${ }^{(19)}$. Em adultos jovens, o efeito do retalho faríngeo sobre as dimensões nasais e nasofaríngeas foi investigado e correlacionado às queixas respiratórias, constatando-se o aparecimento ou o agravamento dessas queixas em $55 \%$ dos casos em curto prazo, e em $36 \%$ em longo prazo. Na avaliação aerodinâmica, o estudo observou redução das dimensões nasofaríngeas mais acentuada nos pacientes que relataram queixas respiratórias somente após a cirurgia, destacando a importância de se acompanhar os resultados cirúrgicos por meio de medidas objetivas ${ }^{(14)}$. Outro estudo, envolvendo crianças, analisou os sintomas respiratórios e investigou as complicações cirúrgicas do retalho faríngeo, identificando dessaturação de oxigênio em $8 \%$ da amostra e achados positivos de apneia obstrutiva do sono em $3 \%$, seis meses após a cirurgia ${ }^{(20)}$.

Em termos de correção da IVF, a cirurgia deve ser realizada o mais precocemente possível, desde que haja boa permeabilidade nasal. Entretanto, os efeitos da técnica de retalho faríngeo sobre a respiração e sua correlação com as dimensões dos orifícios velofaríngeos nas diferentes faixas etárias tem sido assunto pouco explorado até o momento. Somado a isso, deve-se considerar o fato de que as alterações respiratórias relacionadas à obstrução nasofaríngea durante o sono aumentam com a idade ${ }^{(21)}$. O ronco durante o sono, os episódios de apneia obstrutiva do sono e outros sintomas diurnos manifestam-se com maior frequência após os $40 \operatorname{anos}^{(21-23)}$.

Assim, o propósito deste estudo foi verificar a influência da faixa etária sobre as dimensões nasofaríngeas e os sintomas respiratórios em pacientes submetidos à cirurgia para a correção da insuficiência velofaríngea por meio da técnica de retalho faríngeo.

\section{MÉTODOS}

Estudo retrospectivo e transversal que recebeu aprovação do Comitê de Ética em Pesquisa em Seres Humanos do Hospital de Reabilitação de Anomalias Craniofaciais da Universidade de São Paulo (protocolo no 168/2009).

Inicialmente, foram selecionados, por meio de amostragem por conveniência e de acordo com a ordem de atendimento na Instituição, os prontuários de indivíduos que apresentavam fissura palatina e que haviam sido submetidos à cirurgia para a correção de IVF por meio da técnica de retalho faríngeo. Não 
foram incluídos na amostra os casos que apresentavam síndromes, alterações neurológicas e fístula residual no palato, além dos casos que tinham sido submetidos a outros procedimentos cirúrgicos após a colocação do retalho faríngeo e que poderiam interferir na permeabilidade nasal ou nasofaríngea, como a revisão cirúrgica secundária e cirurgias nasais.

Assim, foram selecionados 103 casos e formados três grupos de acordo com a faixa etária: 32 crianças entre 6 e 12 anos (12 do gênero feminino e 20 do gênero masculino), 55 adultos jovens entre 18 e 30 anos ( 31 do gênero feminino e 24 do gênero masculino) e 16 adultos de meia-idade, considerando indivíduos com idade igual ou superior a 40 anos ( 8 do gênero feminino e 8 do gênero masculino). As informações desses casos, quanto à área nasofaríngea e à presença de sinais e sintomas de alteração respiratória, em média 12 meses após a colocação do retalho faríngeo, foram obtidas nos prontuários da instituição.

As dimensões nasofaríngeas foram estimadas pela medida da área dos orifícios velofaríngeos durante a respiração nasal de repouso, por meio da técnica fluxo-pressão, utilizando o sistema computadorizado PERCI-SARS, versão 3.30 (Microtronics Corp®.). Essa técnica baseia-se no princípio de que a área de um orifício pode ser estimada pela medida simultânea da pressão diferencial entre os dois lados do orifício e do fluxo aéreo que passa através deste ${ }^{(24)}$. Assim, durante a realização de vários ciclos respiratórios, um cateter posicionado na cavidade oral e outro em uma das narinas, por meio de um obturador nasal, mensuram as pressões aéreas estáticas, que são transmitidas a transdutores de pressão. Além disso, simultaneamente, um tubo plástico conectado a um pneumotacógrafo aquecido e ligado a um transdutor de pressão é colocado na outra narina, a de maior fluxo, para mensurar o fluxo aéreo nasal. Os sinais dos três transdutores (pressão nasal, pressão oral e fluxo nasal) são enviados ao sistema PERCI para análise por software específico.

$\mathrm{O}$ valor médio de referência utilizado foi de $0,570 \mathrm{~cm}^{2}$, conforme proposto em estudo realizado em adultos normais ${ }^{(25)}$. Sendo assim, áreas menores que $0,570 \mathrm{~cm}^{2}$ foram consideradas como de provável obstrução nasofaríngea.

O levantamento dos sinais e sintomas respiratórios foi realizado a partir de questões de uma ficha de investigação, aplicada por rotina e arquivada no prontuário. As perguntas são baseadas no questionário proposto por Caouette-Laberge et al. ${ }^{(11)}$, sendo consideradas, na análise, as informações referentes à respiração oral diurna e/ou durante o sono, ronco durante o sono e dificuldade respiratória durante o sono. Foram considerados apenas relatos de piora de sintomas pré-existentes ou o aparecimento de queixas respiratórias não relatadas antes da cirurgia.

A significância das diferenças entre os três grupos etários foi verificada por meio de análise de variância a um critério para a área nasofaríngea e por meio do teste do Qui-quadrado para os sintomas respiratórios. Foram aceitos como significativos os valores de $\mathrm{p}<0,05$.

\section{RESULTADOS}

Em relação à área nasofaríngea, a comparação dos valores não mostrou diferença entre os três grupos etários estudados ( $>0,05$ ). Considerando o valor de referência de $0,570 \mathrm{~cm}^{2}$ para a área nasofaríngea, o grupo de crianças e o grupo de adultos de meia-idade apresentaram valores médios abaixo do esperado, ressaltando-se que uma das crianças não foi submetida ao exame rinomanométrico (Tabela 1).

Tabela 1. Valores médios e desvio-padrão da área nasofaríngea $\left(\mathrm{cm}^{2}\right)$ após a cirurgia do retalho faríngeo

\begin{tabular}{lcc}
\hline Grupos & $\begin{array}{c}\text { Área nasofaríngea } \\
\left(\mathrm{cm}^{2}\right)\end{array}$ & $\begin{array}{c}\text { Valor } \\
\text { de } p\end{array}$ \\
\hline Crianças $(\mathrm{n}=31)$ & $0,527 \pm 0,215$ & \\
Adultos jovens $(\mathrm{n}=55)$ & $0,599 \pm 0,213$ & $\mathrm{p}>0,05$ \\
Adultos de meia-idade $(\mathrm{n}=16)$ & $0,488 \pm 0,276$ & \\
\hline
\end{tabular}

Teste ANOVA: Análise de variância a um critério $(p<0,05)$

Quanto ao levantamento dos sintomas respiratórios relatados após a cirurgia, houve relatos de respiração oral, ronco e dificuldades respiratórias durante o sono em todos os grupos estudados. A comparação entre os grupos, para cada sintoma, indicou diferença no que se refere ao ronco, relatado descrito com maior frequência entre os adultos de meia-idade $(\mathrm{p}<0,05)$ (Tabela 2).

\section{DISCUSSÃO}

O estudo do efeito da cirurgia de retalho faríngeo sobre a respiração é de fundamental importância na análise do sucesso cirúrgico desse procedimento. Ainda que muitos estudos tenham relatado seus resultados cirúrgicos exclusivamente

Tabela 2. Frequência relativa e absoluta de sintomas respiratórios após a cirurgia do retalho faríngeo

\begin{tabular}{lccc}
\hline \multirow{2}{*}{ Grupos } & \multicolumn{3}{c}{ Sintomas respiratórios } \\
\cline { 2 - 4 } & Respiração oral & Ronco & Dificuldade respiratória durante o sono \\
\hline Crianças $(n=32)$ & $66 \%(21)$ & $69 \%(22)$ & $9 \%(3)$ \\
Adultos jovens $(n=55)$ & $51 \%(28)$ & $49 \%(27)$ & $7 \%(4)$ \\
Adultos de meia-idade $(n=16)$ & $75 \%(12)$ & $75 \%(12)^{*}$ & $19 \%(3)$ \\
\hline Teste Qui-quadrado & & $\mathrm{p}<0,05$ & \\
\hline
\end{tabular}

*Teste Qui-quadrado indicando maior frequência para os adultos de meia-idade 
quanto aos sintomas da disfunção velofaríngea, a investigação sobre o aparecimento de sintomas respiratórios sempre deve ser valorizada. É sabido que sintomas como ronco e discreta sensação de obstrução nasal são frequentemente observados no pós-operatório imediato da cirurgia de retalho faríngeo, em função da diminuição do espaço nasofaríngeo provocada pelo edema. Entretanto, quando tais sintomas persistem por longo período, agravados por outros, como respiração oral, episódios de obstrução respiratória durante o sono e até mesmo sintomas diurnos como cansaço excessivo, cefaléia e sonolência, o paciente deve ser criteriosamente avaliado.

Sendo assim, a observação e investigação dos sinais e sintomas respiratórios deve caracterizar-se como prática clínica em serviços que fazem uso do retalho faríngeo, avaliando-se rotineiramente, de forma subjetiva e instrumental, os efeitos da cirurgia do retalho faríngeo sobre a respiração ${ }^{(14,26)}$. Partindo desse pressuposto, este estudo pretendeu verificar o efeito da idade sobre as dimensões nasofaríngeas e os sinais e sintomas respiratórios em pacientes com fissura labiopalatina, submetidos à cirurgia de retalho faríngeo, comparando-se os resultados obtidos em três diferentes faixas etárias, uma vez que a cirurgia pode ser indicada para diversas idades, a partir dos seis anos.

Há que se considerar que o ideal é que a cirurgia ocorra o mais precocemente possível para minimizar os sintomas de fala. Entretanto, em função de fatores como condições gerais de saúde, alterações dento-oclusais e presença de articulações compensatórias, a cirurgia poderá ocorrer tardiamente.

Ao comparar-se os valores médios da área nasofaríngea entre os três grupos, não foi encontrada diferença entre eles. Ainda assim, vale ressaltar que valores ligeiramente menores, com média de $0,488 \mathrm{~cm}^{2}$, foram observados no grupo de adultos de meia-idade. Da mesma forma, ao se comparar os valores obtidos ao valor de referência de $0,570 \mathrm{~cm}^{2}$, fica nítida a redução observada nesse mesmo grupo. A área nasofaríngea dos adultos jovens não mostrou indícios de obstrução.

Estudo recente analisou, de forma similar, a relação entre área nasofaríngea e sintomas respiratórios em adultos jovens (média de 20 anos) submetidos ao mesmo procedimento cirúrgico, tendo sido constatada maior frequência de relato de sintomas respiratórios nos pacientes com área nasofaríngea abaixo de $0,550 \mathrm{~cm}^{2}$. Dessa forma, os autores concluíram que valores abaixo de $0,550 \mathrm{~cm}^{2}$ podem estar relacionados a um retalho faríngeo obstrutivo, sendo necessária uma avaliação mais criteriosa ${ }^{(14)}$. Em função da própria natureza do retalho em atuar como uma obstrução mecânica, impedindo o escape da corrente aérea expiratória durante a fala, a redução do espaço nasofaríngeo é um resultado esperado em qualquer idade. Entretanto, essa redução não deve ser excessiva a ponto de desencadear sintomas respiratórios que comprometam a qualidade de vida.

$\mathrm{Na}$ análise da proporção de sintomas respiratórios relatados pelos três grupos etários, foi possível verificar diferença apenas para o sintoma ronco, que ocorreu em $75 \%$ dos adultos de meia-idade, em $69 \%$ das crianças e em $49 \%$ dos adultos jovens. A maior frequência desses sintomas na população mais velha confirma os resultados dos estudos que já estabeleceram consenso sobre a intensificação dos sintomas respiratórios, principalmente o ronco e a apneia obstrutiva do sono, com o decorrer da idade ${ }^{(21-23)}$.

O ronco é um sintoma que pode ser definido como um ruído fricativo originado nos tecidos moles das vias aéreas superiores, decorrente da vibração da úvula e do palato, ocasionalmente da parede lateral faríngea, epiglote e base de língua durante o sono, sendo muito comum nos homens de meia idade e nas mulheres após a menopausa ${ }^{(27)}$. O sintoma está presente em $90 \%$ a $95 \%$ dos casos de Síndrome da Apneia Obstrutiva do Sono (SAOS).

Um estudo verificou a prevalência de distúrbios respiratórios do sono em adultos de meia-idade ( $\geq 40$ anos) submetidos à cirurgia de retalho faríngeo, encontrando sintomas isolados ou combinados, tais como respiração oral, ronco e episódios de obstrução respiratória. Verificou, também, em 53\% dos casos, sintomas diurnos associados, como sonolência excessiva, cefaléia, cansaço constante, hipertensão e obesidade. Os autores do estudo concluíram que o retalho faríngeo pode contribuir para o agravamento dos distúrbios respiratórios em indivíduos nessa faixa etária ${ }^{(19)}$.

Sendo assim, nos pacientes com retalho faríngeo e idade acima de 40 anos, o ronco não deve estar associado somente à presença do retalho, mas também aos efeitos da idade, o que indica a importância de acompanhamento rigoroso para essa população, além de medidas que auxiliem na prevenção do sintoma, ou que contribuam para evitar o seu agravamento, como posição correta ao dormir, controle de peso e circunferência cervical, bem como adoção de hábitos saudáveis.

Ainda que não tenha sido possível observar diferença entre os sintomas de respiração oral e dificuldades respiratórias durante sono entre os três grupos, verificou-se tendência dos adultos de meia-idade a apresentarem maior frequência, principalmente desse último sintoma. A proporção de relato de dificuldade respiratória durante sono em 19\% dos indivíduos é considerada alta do ponto de vista clínico, dada a gravidade do sintoma, pois pode ser um forte indicador de apneia obstrutiva do sono. Entretanto, o exame padrão ouro que pode confirmar tais achados é a polissonografia.

Na literatura, a ocorrência de alterações respiratórias após a faringoplastia é extremamente diversa, variando de $4 \%$ a $92 \%{ }^{(12-14)}$. Tal diversidade pode estar relacionada à metodologia utilizada, idade da população estudada e critérios estabelecidos para definir a presença de alterações respiratórias, levando-se em conta, ainda, a tolerância individual de cada paciente aos sintomas apresentados após a cirurgia.

Outro resultado observado neste estudo foi o fato de as crianças apresentarem maior frequência de sintomas que os adultos jovens. Investigam-se, assim, alguns fatores que possam justificar tal fato, como a presença de maior incidência de infecções de vias aéreas superiores ${ }^{(28)}$ e hipertrofia de tonsilas 
palatinas e faríngea nessa faixa etária ${ }^{(29)}$, o que pode agravar os sintomas respiratórios ${ }^{(30)}$. Além disso, a presença de um cuidador ou responsável por informações sobre esse aspecto, no grupo de crianças, favorece um relato mais detalhado dos sinais e sintomas respiratórios durante o sono, o que não ocorre com muitos adultos jovens. Nesses casos, a ocorrência de tais sintomas pode ser subestimada, uma vez que o sono nesse grupo é pouco observado por outros.

Assim sendo, fica evidente a importância do levantamento dos sinais e sintomas respiratórios associado à avaliação instrumental nos casos submetidos à cirurgia de retalho faríngeo, visto que a avaliação aerodinâmica mostrou-se um indicador de alteração nas dimensões nasofaríngeas e o questionário confirmou a ocorrência de sintomatologia respiratória. Entretanto, somente o exame polissonográfico tem condições de diagnosticar, precisamente, a gravidade das dificuldades respiratórias durante o sono.

Desse modo, a indicação da cirurgia de retalho faríngeo deve continuar sendo realizada o mais precocemente possível, com o objetivo de eliminar ou minimizar os sintomas de fala, bem como as repercussões psicossociais. No entanto, o acompanhamento rigoroso e sistemático desses casos ao longo da vida deve ser uma conduta adotada no controle dos sintomas respiratórios. Além disso, a realização de outros tratamentos cirúrgicos, considerados mais fisiológicos sob o ponto de vista anatômico, ou procedimentos protéticos para correção da insuficiência velofaríngea podem se configurar em alternativas eficientes e preventivas para essa população.

\section{CONCLUSÃO}

A idade, na ocasião da cirurgia de retalho faríngeo, não interferiu nas dimensões nasofaríngeas, mas foi um fator agravante na ocorrência de sintomas respiratórios após a cirurgia, principalmente nos sujeitos mais velhos.

\section{REFERÊNCIAS}

1. Bertier CE, Trindade IEK, Silva Filho OG. Cirurgias primárias de lábio e palato. In: Trindade IEK, Silva Filho OG. Fissuras labiopalatinas: uma abordagem interdisciplinar. São Paulo: Santos; 2007. p. 73-85.

2. Genaro KF, Fukushiro AP, Suguimoto MLFCP. Avaliação e tratamento dos distúrbios da fala. In: Trindade IEK, Silva Filho OG. Fissuras labiopalatinas: uma abordagem interdisciplinar. São Paulo: Santos; 2007. p. 109-22.

3. Kummer AW. Velopharyngeal dysfunction (VPD) and resonance disorders. In: Kummer AW. Cleft palate and craniofacial anomalies: the effects on speech and resonance. San Diego: Singular; 2001. p. 145-76.

4. Peterson-Falzone SJ, Hardin-Jones MA, Karnell MP. Communication disorders associated with cleft palate. In: Peterson-Falzone SJ, Hardin-Jones MA, Karnell MP. Cleft palate speech. $3^{\text {rd }}$ ed. Saint Louis: Mosby; 2001. p.162-98.
5. Rocha DL. Tratamento cirúrgico da insuficiência velofaríngea. In: Trindade IEK, Silva Filho OG. Fissuras labiopalatinas: uma abordagem interdisciplinar. São Paulo: Santos; 2007. p.145-63.

6. Seagle MB, Mazaheri MK, Dixon-Wood VL, Williams WN. Evaluation and treatment of velopharyngeal insufficiency: the University of Florida experience. Ann Plastic Surg. 2002May;48(5):464-70.

7. Berkowitz S. Variations in nasopharyngeal skeletal architecture. In: Berkowitz S. Cleft lip and palate: diagnosis and management. $2^{\text {nd }} e d$. New York: Springer; 2005. p.621-42.

8. Morris HL, Bardach J, Jones D, Christiansen JL, Gray SD. Clinical results of pharyngeal flap surgery: the Iowa experience. Plast Reconstr Surg. 1995Apr;95(4):652-62.

9. Dailey SA, Karnell MP, Karnell LH, Canady JW. Comparison of resonance outcomes after pharyngeal flap and furlow doubleopposing z-plasty for surgical management of velopharyngeal incompetence. Cleft Palate Craniofac J. 2006Jan;43(1):38-43.

10. Fukushiro AP, Trindade IEK. Nasometric and aerodynamic outcome analysis of pharyngeal flap surgery for the management of velopharyngeal insufficiency. J Craniofac Surg. 2011Sep;22(5):164751.

11. Caouette-Laberge L, Egerszegi EP, de Remont AM, Ottenseyer I. Long-term follow-up after division of a pharyngeal flap for severe nasal obstruction. Cleft Palate Craniofac J. 1992Jan;29(1):27-31.

12. Wells MD, Vu TA, Luce EA. Incidence and sequelae of nocturnal respiratory obstruction following posterior pharyngeal flap operation. Ann Plast Surg. 1999Sep;43(3):252-7.

13. Liao YF, Chuang ML, Chen PKT, Chen NH, Yun C, Huang CS. Incidence and severity of obstructive sleep apnea following pharyngeal flap surgery in patients with cleft palate. Cleft Palate Craniofac J. 2002May;39(3):312-6.

14. Yamashita RP, Trindade IEK. Long-term effects of pharyngeal flaps on the upper airways of subjects with velopharyngeal insufficiency. Cleft Palate Craniofac J. 2008Jul;45(4):364-70.

15. Fukushiro AP, Trindade IEK. Nasal airway dimensions of adults with cleft lip and palate: differences among cleft types. Cleft Palate Craniofac J. 2005Jul;42(4): 396-402.

16. Warren DW, Trier WC, Bevin AG. Effect of restorative procedures on the nasopharyngeal airway in cleft palate. Cleft Palate J. 1974Oct; 11:367-73.

17. Smith BE, Skef Z, Cohen M, Dorf DS. Aerodynamic assessment of the results of pharyngeal flap surgery: a preliminary investigation. Plast Reconstr Surg. 1985Sep;76(3):402-10.

18. Witssel DL, Drake AF, Warren DW. Preliminary data on the effect of pharyngeal flaps on the upper airway in children with velopharyngeal inadequacy. Laryngoscope. 1994Jan;104(1):12-5.

19. Trindade IEK, Fukushiro AP, Yamashita RP, Sampaio ACM, Trindade Junior AS. Prevalence of sleep-disordered breathing in middleaged cleft adults with pharyngeal flaps. In: Proceedings of the 10th International Congress on Cleft Palate and Related Craniofacial Anomalies. September 4-8, 2005 Durban, South Africa International Confederation for Cleft Palate and Related Craniofacial Anomalies. 2005: 171 . 
20. Cole P, Banerji S, Hollier L, Stal S. Two hundred twenty-two consecutive pharyngeal flaps: an analysis of postoperative complications. J Oral Maxillofac Surg. 2008Apr;66(4):745-8.

21. Mazzotti DR, Guindalini C, Sosa AL, Ferri CP, Tufik S. Prevalence and correlates for sleep complaints in older adults in low and middle income countries: a 10/66 Dementia Research Group study. Sleep Med. 2012Jun;13(6):697-702.

22. Silva A, Andersen ML, De Mello MT, Bittencourt LR, Peruzzo D, Tufik S. Gender and age differences in polysomnography findings and sleep complaints of patients referred to a sleep laboratory. Braz J Med Biol Res. 2008Dec;41(12):1067-75.

23. Tufik S, Santos-Silva R, Taddei JA, Bittencourt LR. Obstructive sleep apnea syndrome in the Sao Paulo Epidemiologic Sleep Study. Sleep Med. 2010May;11(5):441-6.

24. Warren DW, Dubois AB. A pressure-flow technique for measuring velopharyngeal orifice area during continuous speech. Cleft Palate J. 1964Jan;1:52-71.

25. Sampaio ACM, Trindade IEK, Genaro KF, Yamashita RP, Trindade Junior AS. Dimensões das vias aéreas na respiração de repouso: área nasal e faríngea. I Congresso Científico da USP; 1997 Nov 28-29; Bauru. Livro de Resumos. Bauru: USP; 1997.
26. Zuiani TBB, Trindade IEK, Yamashita RP, Trindade Junior AS. The pharyngeal flap surgery in patients with velopharyngeal insufficiency: perceptual and nasometric speech assessment. Braz J Dysmorphol Speech Disord. 1998;2(1):31-42.

27. Mancini MC, Aloe F, Tavares S. Apnéia do sono em obesos. Arq Bras Endócrinol Metab. 2000Feb;44(1):81-90.

28. Pitrez PMC, Pitrez JLB. Infecções agudas das vias aéreas superiores - diagnóstico e tratamento ambulatorial. J Pediatr. 2003;79(Supl.1):77-86.

29. Bonuck KA, Chervin RD, Cole TJ, Emond A, Henderson J, Xu $\mathrm{L}$ et al. Prevalence and persistence of sleep disordered breathing symptoms in young children: a 6-year population-based cohort study. Sleep. 2011Jul;34(7):875-84.

30. Izu SC, Itamoto $\mathrm{CH}$, Pradella-Hallinan M, Pizarro GU, Tufik S, Pignatari S et al. Ocorrência da síndrome da apneia obstrutiva do sono (SAOS) em crianças respiradoras orais. Braz J Otorhinolaryngol. 2010;76(5):552-6. 